\title{
Null Double Injection and the Extra Element Theorem
}

\author{
R. D. MIDDLEBROOK, FELLOW, IEEE
}

\begin{abstract}
The extra element theorem (EET) states that any transfer function of a linear system can be expressed in terms of its value when a given "extra" element is absent, and a correction factor involving the extra element and two driving point impedances seen by the element. One class of applications is when a system has already been analyzed and later an extra element is to be added to the model: the EET avoids the analysis having to be restarted from scratch. Another class of applications is when a system is to be analyzed for the first time: if one element is designated as "extra," the analysis can be performed on the simpler model in the absence of the designated element, and the result modified by the EET correction factor upon restoration of the "extra" element.

Although the EET itself is not new, its interpretation and application appear to be little known. In this paper, the EET is derived and applied to several examples in a manner that has been developed and refined in the classroom over a number of years. The concept of "null double injection" is introduced first, because it is the key to making easy the calculation of the two driving point impedances needed for the EET correction factor.
\end{abstract}

\section{INTRODUCTION}

$I^{2}$ $\mathrm{N}$ a design process, considerable effort is expended in analysis of a circuit model for various transfer functions, of which usually the gain is the most important. Later, perhaps following experimental measurements on a breadboard, it may be decided that an additional element needs to be added to the model. For example, gain measurements may disclose an unanticipated high-frequency pole, and one may wish to restore a previously omitted collector-base capacitance to a transistor model.

The usual approach is to restart the analysis on the augmented circuit model, for all the desired transfer functions. Each such analysis is of course more complicated than it was the first time, especially if there is feedback to be accounted for, because the circuit has an additional element.

In this iteration process, it may be noticed that much of the analysis is the same as it was the first time. One would like to avoid repeating the parts that are the same, and do only the parts that are different because of the presence of the additional element.

The extra element theorem provides this shortcut. The gain (or any other transfer function) in the presence of an extra element is given by a correction factor multiplying

Manuscript received October 20, 1988.

The author is with the Department of Electrical Engineering, California Institute of Technology, Pasadena, CA 91125.

IEEE Log Number 8929054. the gain already calculated in the absence of the extra element. The correction factor contains only the extra element itself and two driving point impedances, both calculated at the place where the extra element is to be added, but under two different conditions. Thus, all the work done on the original circuit is utilized and only the two driving point impedances "seen" by the extra element need to be determined. Moreover, the correction-factor format itself is useful because it exposes directly the modification to the original result caused by the extra element.

The extra element theorem is of benefit if calculation of the two driving point impedances is easier, or is less work, than calculation of the gain directly from the augmented circuit. While there is no guarantee, this is usually so, and leads to usefulness of the theorem in another context.

Since the gain and the two driving point impedances are calculated for the circuit in the absence of the extra element, no calculations are done on the augmented circuit itself; one merely inserts the extra element into the correction factor. This affords a method of obtaining transfer function results by analysis only of a circuit that is less complicated by one element. Thus, in analysis of a given circuit, one may designate an element as "extra" and omit it, perform the analysis for the desired transfer functions and driving-point impedances on the simpler "reference" circuit, and then restore the missing element by the extra element theorem correction factor. It will be seen in the examples to follow that this approach is particularly simple and illuminating when the reference circuit is resistive and the extra element is a reactance, since then the driving point impedances are resistive and the correction factor gives directly the pole and zero due to the reactance.

There are several ways of establishing the extra element theorem. However, since one of the driving point impedances involves a null double injection condition, I have found it pedagogically preferable to introduce null double injection first, as is done here, since this concept is also useful for other purposes. Indeed, since most students have not previously heard of null double injection, most of the time spent on the extra element theorem is devoted to convincing them, through practice, that null double injection calculations are always simpler than single injection calculations; it is only because the concept is unfamiliar that the null double injection calculations appear at first to be more difficult. 


\section{Null Double Injection}

Consider a linear system with two driving signals $u_{i 1}$ and $u_{i 2}$, as indicated schematically in Fig. 1. These driving signals can be voltage, current, torque, or any independent "inputs."

These two "input" signals cause the various branch currents, node voltages, etc. to take on certain values. If the system is linear, the only constraint to be placed upon it, each such dependent signal, by superposition, is the linear sum of the two values it would have due to each input signal alone.

Let two dependent signals be designated as "outputs" $u_{o 1}$ and $u_{o 2}$. Thus,

$$
\begin{aligned}
& u_{o 1}=A_{1} u_{i 1}+A_{2} u_{i 2} \\
& u_{o 2}=B_{1} u_{i 1}+B_{2} u_{i 2}
\end{aligned}
$$

where the coefficients are transfer functions, or "gains," formally defined as

$$
\begin{aligned}
& \left.\frac{u_{o 1}}{u_{i 1}}\right|_{u i 2=0} \equiv A_{1} \\
& \left.\frac{u_{o 1}}{u_{i 2}}\right|_{u_{i 1}=0} \equiv A_{2} \\
& \left.\frac{u_{o 2}}{u_{i 1}}\right|_{u_{i 2}=0} \equiv B_{1} \\
& \left.\frac{u_{o 2}}{u_{i 2}}\right|_{u_{i 1}=0} \equiv B_{2} .
\end{aligned}
$$

Usually, only one input is considered at a time, and the system is analyzed separately for the transfer functions $A_{1}, B_{1}$ and $A_{2}, B_{2}$. However, some additional useful results can be obtained by application of both inputs simultaneously, which is the condition of "double injection."

At first sight, it may seem that double injection is of little use since either output can have any value. The usefulness appears, however, when one output is made to have the specific value zero, which is the condition of null double injection.

Let output number 1 be nulled. Then (1) and (2) become

$$
\begin{aligned}
0 & =\left.A_{1} u_{i 1}\right|_{u_{o 1}=0}+\left.A_{2} u_{i 2}\right|_{u_{o 1}=0} \\
\left.u_{o 2}\right|_{u_{o 1}=0} & =\left.B_{1} u_{i 1}\right|_{u_{i \mid}=0}+\left.B_{2} u_{i 2}\right|_{u_{o l}=0} .
\end{aligned}
$$

A specific ratio of the two inputs, namely

$$
\frac{u_{i 2}}{u_{i 1}}=\left.\frac{u_{i 2}}{u_{i 1}}\right|_{u_{i 1}=0}
$$

is needed to null $u_{o l}$, and a specific ratio of output number 2 to either input results, as indicated by the constraints in (7) and (8).

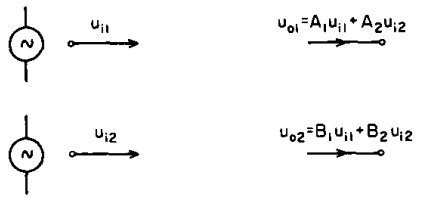

Fig. 1. Model of a linear system with two inputs and two outputs

By elimination of $\left.u_{i 1}\right|_{u_{i 1}=0}$ from (7) and (8)

$$
\left.\frac{u_{o 2}}{u_{i 2}}\right|_{u_{01}=0}=\frac{A_{1} B_{2}-A_{2} B_{1}}{A_{1}} .
$$

Note the difference from (6), repeated here:

$$
\left.\frac{u_{o 2}}{u_{i 2}}\right|_{u_{i 1}=0}=B_{2} \text {. }
$$

The algebra of the above steps is simple. The important thing is the physical interpretation of the null double injection condition and the understanding of why the two results of (10) and (11) are different. I use a hardware demonstration to illustrate the above sequence of steps. The two input sine waves and the two output sine waves are observed on a four-trace scope.

First, with $u_{i 2}$ zero, $u_{i 1}$ is varied up and down in amplitude, and both $u_{o 1}$ and $u_{o 2}$ vary up and down in (different) proportions, corresponding to the two gains $A_{1}$ and $B_{1}$. The process is repeated with $u_{i 2}$ alone, and the (same) two outputs vary up and down in different proportions representing the gains $A_{2}$ and $B_{2}$.

With $u_{i 2}$ fixed at some arbitrary value, together with the corresponding values of $u_{o 1}$ and $u_{o 2}$, an arbitrary value of $u_{i 1}$ is simultaneously applied: $u_{o 1}$ and $u_{o 2}$ suddenly change to different values, representing the superposition of (1) and (2).

Now, with one input remaining fixed in amplitude, the other is varied; both outputs vary. At some value of the variable input one of the outputs goes through a null (if it doesn't, the phase of one input is reversed and the search repeated). Once the null at one output has been achieved, the other output takes on a fixed value. Conditions are now as expressed in (7) and (8).

Several other points can now be demonstrated. Either or both inputs can be varied to establish the null; since the null is determined solely by their ratio, (9), the two inputs can be varied up or down in proportion, and the null remains. At the same time, the output which is not nulled varies up and down in proportion to either input, and it can easily be seen that its ratio to one input, (10), is not the same as when the other input is zero, (11).

In setting up this demonstration, one requires both inputs to be sine waves of the same frequency with fixed phase relationship, and one therefore derives both from the same oscillator. If the test circuit is purely resistive, one input can be derived from the other by a potentiometer and reversing switch since only in-phase or out-ofphase signals are required to achieve a null. However, if 
the test circuit is reactive, a null cannot be obtained merely by adjustment of the amplitude of one input with respect to the other and a phase adjustment is also required. For convenience in demonstration, independent magnitude and phase adjustments are desired, and it is an instructive laboratory project for a student to determine how to obtain a wide-range phase adjustment independent of frequency and with constant amplitude output.

Although the experimental demonstration is helpful to students in understanding the physical meaning, null double injection is valuable principally as an analytical tool. At this stage, its value is not yet clear; it lies in the fact that the transfer function expressed by $(10)$ is both physically significant and useful in its own right, and easy to calculate for a given circuit. Its applications lie in sensitivity theory, feedback theory, and in the extra element theorem to be developed in the next section.

\section{The Extra Element Theorem (EET) for a Parallel Element}

The extra element theorem (EET) is developed from a sequence of two special cases of the pair of transfer functions given by (10) and (11).

First, identify the input number 2 as a current $u_{i 2}=i$ and output number 2 as a voltage $u_{o 2}=v$, as in Fig. 2 .

Equations (1) and (2) become

$$
\begin{gathered}
u_{o 1}=A_{1} u_{i 1}+A_{2} i \\
v=B_{1} u_{i 1}+B_{2} i
\end{gathered}
$$

and (10) and (11) become

$$
\begin{aligned}
& \left.\frac{v}{i}\right|_{u_{01}=0}=\frac{A_{1} B_{2}-A_{2} B_{1}}{A_{1}} \\
& \left.\frac{v}{i}\right|_{u_{i 1}=0}=B_{2} .
\end{aligned}
$$

The transfer functions $v /\left.i\right|_{u_{\nu 1}=0}$ and $v /\left.i\right|_{u_{i 1}=0}$ are now identified as transimpedances.

Second, identify the output voltage $v$ as being the voltage across the terminals at which the current $i$ is injected, as in Fig. 3. The two transimpedances now become selfimpedances interpreted as driving point impedances $Z_{d p}$ "seen" by the second input:

$$
\begin{aligned}
& \left.\frac{v}{i}\right|_{u_{o l}=0}=\left.Z_{d p}\right|_{u_{o l}=0} \equiv Z_{n}=\frac{A_{1} B_{2}-A_{2} B_{1}}{A_{1}} \\
& \left.\frac{v}{i}\right|_{u_{i 1}=0}=\left.Z_{d p}\right|_{u_{i 1}=0} \equiv Z_{d}=B_{2} .
\end{aligned}
$$

In the final step, consider the linear system with an impedance $Z$ in place of the second input $u_{i 2}$, as in Fig. 4. The system does not "know" whether the entering current $i$ is an independent signal or not; (1) and (2) still hold. However, there is now an externally imposed rela-

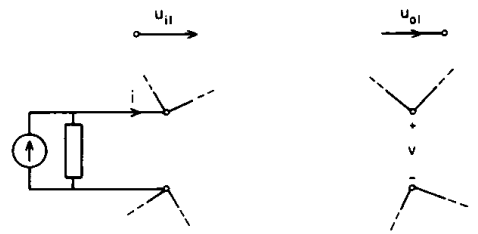

Fig. 2. As Fig. 1, with second input a current $i$ and second output a volt age $v$

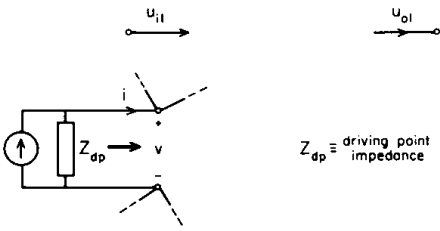

Fig. 3. As Fig. 2, with second output at the same node pair as the second input.

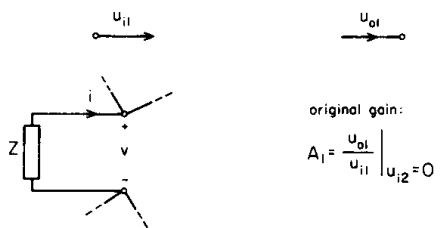

Fig. 4. As Fig. 3, with second output and second input related by an extra impedance $Z$.

tionship between $u_{i 2}=i$ and $u_{o 2}=v$, namely $i=-v / Z$ by Ohm's law. Equations (1) and (2) then become

$$
\begin{gathered}
u_{o 1}=A_{1} u_{i 1}-\frac{A_{2}}{Z} v \\
v=B_{1} u_{i 1}-\frac{B_{2}}{Z} v .
\end{gathered}
$$

By elimination of $v$ :

$$
\frac{u_{o 1}}{u_{i 1}}=A_{1} \frac{1+\frac{1}{Z} \frac{A_{1} B_{2}-A_{2} B_{1}}{A_{1}}}{1+\frac{1}{Z} B_{2}}
$$

which, by substitution of (16) and (17), becomes

$$
\frac{u_{o 1}}{u_{i 1}}=A_{1} \frac{1+\frac{Z_{n}}{Z}}{1+\frac{Z_{d}}{Z}} .
$$

According to the following interpretation, this result is the extra element theorem. In the absence of $Z$ (that is, $Z$ $=\infty$ ) neither the second input nor the second output need be identified, and $u_{i 1}$ and $u_{o 1}$ become the single system "input" and "output." So, by (3), $A_{1}$ is identified as the system transfer function, or gain, $\left.A\right|_{z=\infty}=A_{1}$. 
In (21), $u_{o 1} / u_{i 1}$ is the system transfer function, or gain, after the element $Z$ has been connected across some node pair, and can be identified as $\left.A\right|_{z}$. Hence, (21) can be written

$$
\left.A\right|_{Z}=\left.A\right|_{Z=\infty} \frac{1+\frac{Z_{n}}{Z}}{1+\frac{Z_{d}}{Z}} .
$$

which is the extra element theorem in its final form, and says that the gain of a linear system in the presence of an element $Z$ is the gain of the system when $Z$ is infinite, multiplied by a correction factor involving the extra element $Z$ itself, and two driving point impedances $Z_{n}$ and $Z_{d}$ seen by the extra element. The subscripts $n$ and $d$ refer simply to the "numerator"' and "denominator" positions of the driving point impedances in the correction factor.

If the gain $A \mid Z=\infty$ of the original, or reference, system has already been calculated then, as described in the Introduction, the EET has value if it is easier to calculate the two driving point impedances (on the reference circuit) than it is to calculate the gain $\left.A\right|_{z}$ directly on the circuit augmented by the extra element.

\section{Example 1: Inclusion of Emitter Bypass Capacitance to Common-Emitter Amplifier Stage}

Suppose the basic common-emitter (CE) amplifier stage of Fig. 5 has been designed. A suitable ac small-signal model is shown in Fig. 6, with the transistor replaced by a simple $T$ model in which the nonlinear emitter diode slope resistance $r_{E} \equiv(k T / e) / I_{E}$. The base bias divider is replaced by $R_{B} \equiv R_{1} \| R_{2}$, and the coupling capacitance $C_{1}$ is taken to be an ac short. However, the emitter bypass capacitance is omitted.

Straightforward analysis of the model of Fig. 6 leads to the following result for the gain $A_{0} \equiv v_{2} / v_{1}$ :

$$
A_{0}=\frac{R_{B}}{R_{s}+R_{B}} \frac{\alpha R_{L}}{R+r_{E}+\left(R_{s} \| R_{B}\right) /(1+\beta)}
$$

where $\alpha \equiv \beta /(1+\beta)$.

Suppose now that one wishes to account for the effect of the emitter bypass capacitance $C_{2}$, to be added across the external emitter resistance $R$. Instead of recalculating the entire circuit, let us use the EET to find a correction factor on the gain $A_{0}$ already determined. Thus, the model of Fig. 6 is now identified as the "reference"' circuit, and $A_{0}$ is the "reference gain" corresponding to $\left.A\right|_{Z=\infty}$ where $Z$ is the impedance to be added across $R$.

To find the correction factor, the two driving point impedances across $R$ are to be calculated.

Fig. 7 shows the circuit model modified for the conditions under which $Z_{d}$ is to be calculated: a "second" or "test"' input-signal is applied across $R$, and the "original" input $v_{1}$ is made zero, since $Z_{d} \equiv[v / i]_{p^{\prime}=0}$. In this simple example, the result can be written by inspection:

$$
Z_{d}=R_{d} \equiv R \|\left[r_{E}+\left(R_{s} \| R_{B}\right) /(1+\beta)\right]
$$

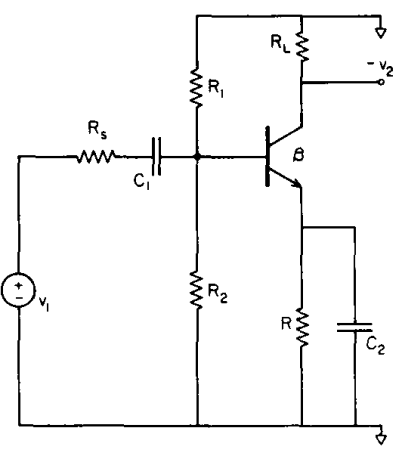

Fig. 5. Basic common-emitter transistor amplifier stage.

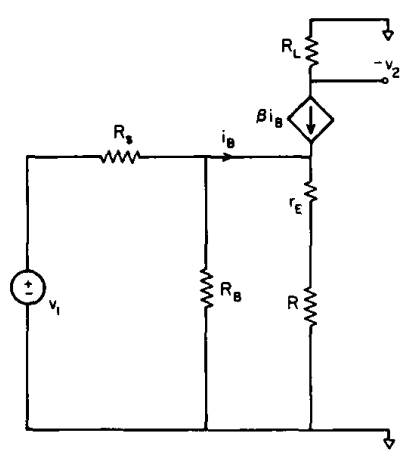

Fig. 6. Simple small-signal ac model of amplifier of Fig. 5, for calculation of gain $v_{2} / v_{1}$ with $C_{1}$ taken as short and $C_{2}$ as open.

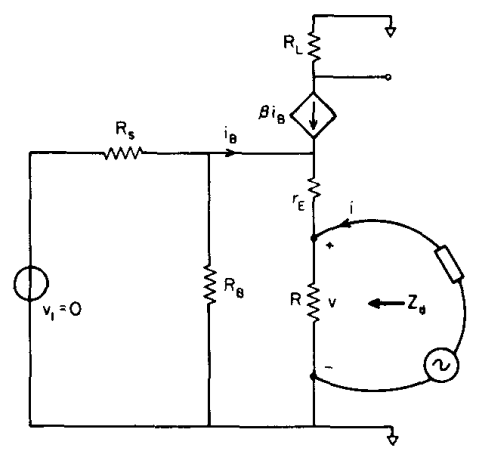

Fig. 7. Conditions for calculation of the single-injection driving point impedance $Z_{d l}$ seen by $C_{2}$ as an "extra" element in the model of Fig. 6 .

The test signal source can be a voltage or a current source, whichever makes the calculation easier; here, since the answer is obviously the parallel combination of the impedances looking into $R$ and $R_{E}$, the test signal is shown as a general Thevenin source.

Fig. 8 shows the circuit model modified for the conditions under which $Z_{n}$ is to be calculated: a test signal is again applied across $R$, and the original input $v_{1}$ is restored. Thus, double injection is established, and the two inputs are imagined to be mutually adjusted until the original output $v_{2}$ is nulled. Then, $Z_{n} \equiv[v / i]_{t^{\prime 2}=0}$ is the driving point impedance at the test signal input under this null double injection condition. 


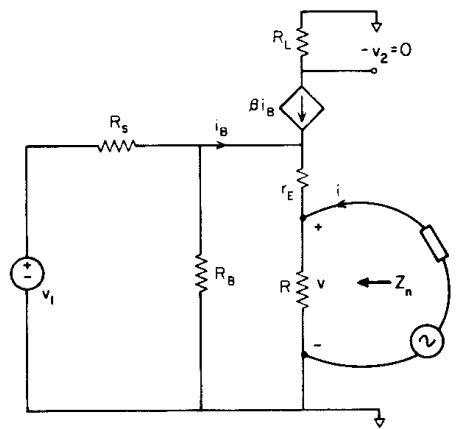

Fig. 8. Conditions for calculation of the null double injection driving poin impedance $Z_{n}$ seen by $C_{2}$ as an "extra" element in the model of Fig. 6.

The following logical sequence of steps leads to the result. Output voltage $v_{2}=0 \Rightarrow$ current in $R_{L}=0 \Rightarrow$ collector current $=0 \Rightarrow$ emitter current $=0$. Therefore, all of the current $i$ from the test source goes through $R$, so

$$
Z_{n}=R_{n} \equiv R \text {. }
$$

Student reaction at this point is usually one of surprise that the answer is so simple. They understand the steps, but that is not enough, and so I have found it useful to illustrate this process with overhead projection overlays. The reference circuit of Fig. 6 is the "base" picture, and an overlay is added that shows, by trace width, a qualitative representation of the relative magnitudes of the currents in the various branches that result from the original input voltage $v_{1}$. The overlay, Fig. 9 , is in a solid blue color, and shows the signal distributions that correspond to the original conditions from which the reference gain $A_{0}$ of (23) are calculated.

The blue overlay is removed, and replaced by a yellow one that shows the currents that result from the test signal alone, as in Fig. 10, and represents the signal distributions from which the single-injection driving point impedance $Z_{d}$ is calculated. The driving point current clearly divides into two paths and, for typical numbers, the bulk of it goes into the $r_{E}$ branch.

Finally, the blue and yellow overlays are superimposed simultaneously upon the reference circuit. This cannot be illustrated here, but with the overhead projection a rather dramatic result is achieved: the trace representing the current through $R_{L}$ turns green, which is identified as the null resulting from cancellation, at the output, of the signals separately proportional to each of the inputs.

It is also seen that several of the branch currents are green, which means that the null "propagates": in particular, because of the properties of the transistor $T$ model, if one current is zero, then all three terminal currents are zero. In the present example, nulled collector current implies nulled emitter current, which leads immediately to the conclusion that all the injected current from the test source goes through $R$, so that $Z_{n}=R$.

Useful Observation 1): To calculate the null driving point impedance $Z_{n}$, see how the null propagates to the

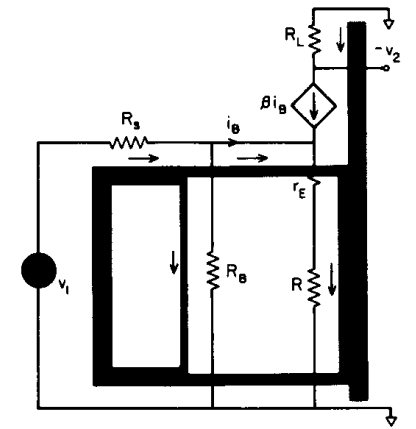

Fig. 9. Qualitative representation of the current distribution in the model of Fig. 6, for calculation of the gain $v_{2} / \nu_{1}$ in the absence of the "extra" element $C_{2}$.

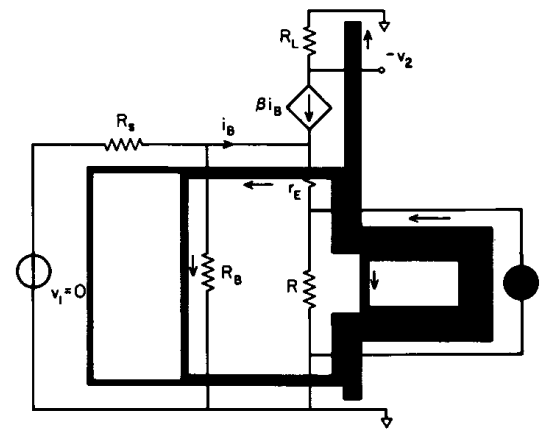

Fig. 10. Qualitative representation of the current distribution in the model of Fig. 6, for calculation of the driving point impedance $Z_{d}$ seen by the "extra" element $C$.

vicinity of the test signal source. Students having initial difficulty doing this may find it helpful to visualize the superposition of signals from the two sources, as illustrated by the overhead projection overlays.

Although unnecessary for calculation of $Z_{n}$, it is helpful to students to notice also the parts of the superimposed blue and yellow overlays that are not green. A separate overlay shows only these nonzero currents, which constitute two separate loop currents, one through $v_{1}, R_{s}$, and $R_{B}$, and the other through the test source and $R$. The absence of current (the null) in all three legs of the transistor model clearly illustrate that the null is achieved by balancing the base-to-ground voltage across $R_{B}$, determined by $v_{1}$, against the emitter-to-ground voltage across $R$, determined by the test source.

Useful Observation 2): To calculate the null driving point impedance $Z_{n}$, it is never necessary to know the relation between the two signal sources. The existence of the null is used instead: the process of null achievement by mutual adjustment of the two sources is irrelevant, and the fact that the null exists is equivalent information. Thus, in the example under discussion, it was not necessary to know $v_{1}$ to find $Z_{n}$.

Useful Observation 3): It is easier to calculate $Z_{n}$ than $Z_{d}$ because the null propagates, so there are fewer contributions to the result, and the answer is simpler. Students 
initially tend to disbelieve this statement, because the concept of single injection ( to find $Z_{d l}$ ) is familiar, whereas the concept of null double injection (to find $Z_{n}$ ) is not; however, with practice, they eventually realize that null double injection is a simple yet powerful technique.

It is now but a short step to complete the example with addition of the extra element, the emitter bypass capacitance $Z \equiv 1 / s C_{2}$. By the EET of (22),

$$
\left.A\right|_{Z}=A_{0} \frac{1+s C_{2} R_{n}}{1+s C_{2} R_{d}}
$$

where $\left.A\right|_{Z=\infty}=A_{0}$ from (23), and $R_{d}$ and $R_{n}$ have been found in (24) and (25).

Useful Observation 4): If the reference circuit is resistive and the extra element is a reactance, the EET correction factor gives directly the pole and/or zero due to the reactance. This is because both driving point impedances, which are calculated on the reference circuit, are resistive in this case.

Useful Observation 5): The extra element itself need not be identified until the last step of substitution into the $E E T$ correction factor. Only its future position needs to be identified since all the other quantities $\left.A\right|_{z=\infty}, Z_{d}$, and $Z_{n}$ are calculated on the reference circuit.

\section{The Extra Element Theorem (EET) for A SERIES ElemENT}

Since the extra element theorem of the previous section permits addition of an extra element across a node pair, it is to be expected that a similar, or dual, form of the theorem would permit addition of an extra element in series with a branch. Such an alternative form need not be derived independently, but can easily be obtained from the original form.

Let $A$ again represent any transfer function of a linear system, such as the gain. The EET for an extra parallel element $Z$ is

$$
\left.A\right|_{Z}=\left.A\right|_{Z=\infty} \frac{1+\frac{Z_{n}}{Z}}{1+\frac{Z_{d}}{Z}}
$$

which can be written

$$
\begin{aligned}
\left.A\right|_{Z}=\left.A\right|_{Z=\infty} \frac{\frac{Z_{n}}{Z} \frac{Z}{Z_{d}} \frac{Z}{Z}+1}{\frac{Z}{Z_{d}}+1} \\
=\left[\left.A\right|_{Z=\infty} \frac{Z_{n}}{Z_{d}}\right] \frac{1+\frac{Z}{Z_{n}}}{1+\frac{Z}{Z_{d}}} .
\end{aligned}
$$

What is the interpretation of the quantity in the square bracket? It must be the gain of the system when $Z=0$ since the remaining factor goes to unity when $Z$ goes to zero. Hence, the dual form of the EET is

$$
\left.A\right|_{Z}=\left.A\right|_{Z=0} \frac{1+\frac{Z}{Z_{n}}}{1+\frac{Z}{Z_{d}}}
$$

which says that the gain of a linear system in the presence of an element $Z$ is the gain of the system when $Z$ is zero, multiplied by a correction factor involving the extra element $Z$ itself, and two driving point impedances $Z_{n}$ and $Z_{d}$ seen by the extra element.

These are the same words that describe the original EET; the difference is that the reference circuit has $Z=$ 0 instead of $Z=\infty$, and the correction factor provided by the theorem has the $Z$ terms inverted. The two driving point impedances $Z_{n}$ and $Z_{d}$ are calculated in the same way.

\section{Example 2: Inclusion of Bypassed Emitter Resistance to Common-Emitter Amplifier Stage}

Consider the same CE amplifier stage of Fig. 5 from a different point of view. At "midband" frequencies where both capacitive reactances are negligible, the ac smallsignal model is as in Fig. 11 , which is the reference circuit. The reference gain is the midband gain $A_{m} \equiv v_{2} / v_{1}$ :

$$
A_{m}=\frac{R_{B}}{R_{s}+R_{B}} \frac{\alpha R_{L}}{r_{E}+\left(R_{s} \| R_{B}\right) /(1+\beta)} .
$$

Suppose now that one wishes to account for the gain change at lower frequencies caused by increasing reactance of $C_{2}$ : the emitter bias resistance $R$ is no longer completely bypassed, and so a nonzero impedance $Z$ needs to be added in series with $r_{E}$.

The EET for an extra series impedance $Z$, (30), can now be invoked, and the two driving point impedances seen by the extra element are calculated on the reference circuit.

Conditions for calculation of $Z_{d}$ are shown in Fig. 12 . A test input signal is applied in series with $r_{E}$, and the original input $v_{1}$ is made zero. Again, the result can be written by inspection:

$$
Z_{d}=R_{d}^{\prime}=r_{E}+\left(R_{s} \| R_{B}\right) /(1+\beta) .
$$

A prime is used to distinguish this result from that in the previous example.

Conditions for calculation of $Z_{n}$ are shown in Fig. 13. The original input $v_{1}$ is restored, and mutually adjusted together with the test signal to set up a null at the original output $v_{2}$. Null double injection conditions are thus established, and the same logical sequence of steps is followed as in the previous example. The null propagates, and nulled $v_{2}$ implies nulled emitter current. This time, however, the emitter current is the only current through the test source, so

$$
Z_{n}=R_{n}^{\prime}=\infty
$$

since a zero $i$ results from nonzero $v$ at the test source driving point. 


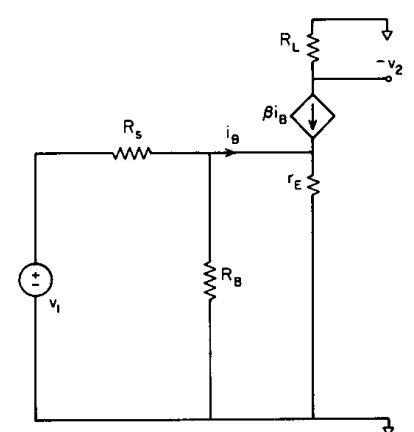

Fig. 11. Small-signal ac model of amplifier of Fig. 5, with both $C_{1}$ and $C_{2}$ taken as shorts

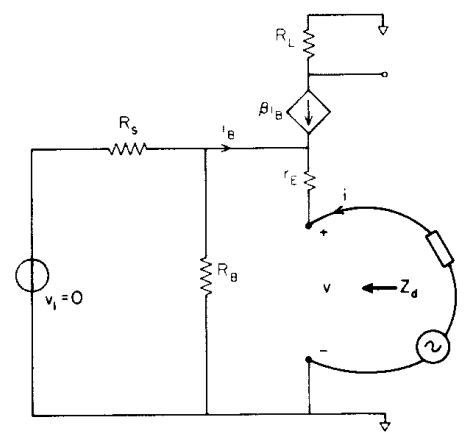

Fig. 12. Conditions for calculation of $Z_{d}$ seen by $C_{2}$ and $R$ in the model of Fig. 11

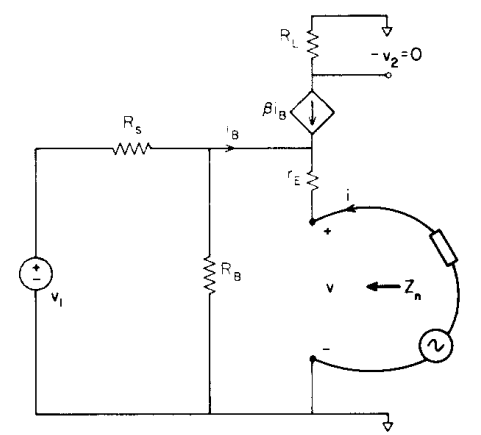

Fig. 13. Conditions for calculation of $Z_{n}$ seen by $C_{2}$ and $R$ in the model of Fig. 11 .

With $Z_{d}$ and $Z_{n}$ now determined, the extra element itself is identified as the parallel combination $R \|\left(1 / s C_{2}\right)$; and insertion into the EET of (30) gives

$$
\begin{aligned}
\left.A\right|_{Z} & =A_{m} \frac{1}{1+\frac{Z}{R_{d}^{\prime}}} \\
& =A_{m} \frac{1+\frac{1}{s C_{2} R}}{1+\frac{1}{s C_{2}\left(R \| R_{d l}^{\prime}\right)}} .
\end{aligned}
$$

However, $R \| R_{d}^{\prime}=R_{d}$ by (24), and $R=R_{n}$ by (25), so

$$
\left.A\right|_{Z}=A_{m} \frac{1+\frac{1}{s C_{2} R_{n}}}{1+\frac{1}{s C_{2} R_{d}}} .
$$

Useful Observation 6): The "extra element"' $Z$ can be any two-terminal combination of elements.

The two results, (26) and (36), are the same since both describe the circuit of Fig. 5 with the bypass capacitance $C_{2}$ in place, but the coupling capacitance $C_{1}$ still considered an ac short. Equation (26) gives the gain with the zero-frequency gain $A_{0}$ as reference, modified by a normal pole and zero; (36) gives the gain with the midband-frequency gain $A_{m}$ as reference, modified by an inverted pole and zero.

Example 2 was chosen specifically to demonstrate that the extra element need not be a single element. A third starting point that leads to the same result illustrates another feature of the EET.

\section{Example 3: Extension of Example 2}

Identify the extra element $Z \equiv 1 / s C_{2}$, as in example 1 , so that $Z_{d}$ and $Z_{n}$ are determined from Figs. 7 and 8 , respectively, with the results of (24) and (25). However, take the reference value to be $Z=0$ instead of $Z=\infty$ : the reference circuit is now as in Fig. II (imagine the emitter resistance $R$ to be present, but shorted out by the reference value $Z=0$ ). Therefore, the reference gain is $\left.A\right|_{Z=0}=A_{m}$ of (31), and the appropriate form of the EET is (30) for the added series element. Substitution of $Z_{d}$ and $Z_{n}$ from (24) and (25) and $A_{m}$ from (31) leads directly to (36).

Useful Observation 7), extension of 4): Either the zero or the infinite frequency gain can be chosen as the reference gain, by choice of $Z=\infty$ or $Z=0$ as reference value for a capacitance, vice versa for an inductance, as extra element. The frequency response due to $Z$ is then in terms of a normal pole and zero modifying the zero frequency gain, or an inverted pole and zero modifying the infinite frequency gain.

In summary, with respect to the original "input" and "output" signals $u_{i 1}$ and $u_{o 1}$, the extra element theorem states that

$$
\frac{\text { "output" }}{\text { "input" }} \equiv A=\left\{\begin{array}{r}
1+\frac{Z_{n}}{Z} \\
\left.A\right|_{Z=\infty} \frac{Z_{d d}}{Z} \\
\left.A\right|_{Z=0} \frac{1+\frac{Z}{Z_{n}}}{1+\frac{Z}{Z_{d}}}
\end{array}\right.
$$


where $Z$ is an "extra" element inserted across a node pair, (23), or in series with a branch, (24), and $Z_{d}$ and $Z_{n}$ are the driving point impedances seen by $Z$, defined by

$$
\begin{array}{ll}
\left.Z_{n} \equiv Z_{d p}\right|_{u_{u 1}=0} & \text { (null double injection) } \\
\left.Z_{d} \equiv Z_{d p}\right|_{u_{i 1}=0} \quad \text { (single injection) } .
\end{array}
$$

Useful Observation 8): Nulling is not the same as shorting (of a voltage). The distinction is important: shorting a node pair changes the system determinant; nulling a voltage across a node pair (or a current through a branch) does not. Wrong answers for $Z_{n}$ are often obtained by students not yet clear about this distinction, and they need to be led through several examples of the "null propagation" process to appreciate at which step their logical error occurs.

A useful first example for students to work out, in class, is to account for the frequency response due to the coupling capacitance $C_{1}$ in the CE amplifier stage of Fig. 5, in which so far $C_{1}$ has been taken to be an ac short. The bypass capacitance $C_{2}$ can be taken either as an open or as a short throughout the exercise, by selection of either Fig. 6 or Fig. 11 as reference circuit. The reference value of $Z \equiv 1 / s C_{1}$ is zero, and the test signal is inserted in series with $R_{s}$ in place of $C_{1}$. Students now derive $Z_{d}$, which (for $C_{2}$ short) is

$$
Z_{d}=R_{d}=R_{s}+R_{B} \|(1+\beta) r_{E} .
$$

A common mistake is to place the two contributing terms in parallel instead of in series.

The result for $Z_{n}$ is

$$
Z_{n}=\infty
$$

the derivation of which often causes mental distress. The "null propagation"' logical sequence of steps is: output voltage $v_{2}=0 \Rightarrow$ current in $R_{L}=0 \Rightarrow$ collector current $=0 \Rightarrow$ base current $=0$. Also, emitter current $=0 \Rightarrow$ voltage across $R_{B}=0 \Rightarrow$ current through $R_{B}=0$. Therefore, since both the base current and the current through $R_{B}$ are zero, there is zero current through the test signal source regardless of its voltage, so $Z_{n}=\infty$. The most common mistake is forgetting that both emitter and base currents are zero if the collector current is zero, an error which leads to the incorrect result $Z_{n}=R_{s}+R_{B}$ or $Z_{n}=$ $R_{s}+(1+\beta) r_{E}$.

Students can be recommended to visualize the superposition of currents due to the two sources under the nulled output condition. It becomes immediately clear that since the two sources are in direct series, the only way that a nulled output can exist is for all signals to be zero, that is, the null is established by adjustment of the two sources to be equal and opposite.

The final step of substitution into the EET for the series extra element, (38), leads to

$$
\left.A\right|_{1 / s C_{l}}=A_{m} \frac{1}{1+\frac{1}{s C_{1} R_{d}}}
$$

with $R_{d}$ given by (41).

\section{The Extra Element Theorem as an Analysis TOOL}

Although the original motivation for the EET was to allow building on a previous result when a new element is to be added, it can also be useful in shortening and simplifying a derivation of a given complete circuit. One of the elements is designated as "extra," and is omitted. Analysis is done for the transfer function of interest, and for the two driving point impedances where the element was removed. Finally, the element is replaced and the correction factor for the previous result is given by the EET.

In this approach, one calculation on the complete circuit is replaced by three calculations on a simpler circuit, with one fewer elements. Of course, there is no guarantee that there will be any benefit in using the EET in this way, but it does have the advantage that the effect of the selected "extra" element is illuminated in the bilinear form of the correction factor. As already mentioned, this is particularly helpful when the selected element is the only reactance, since then the EET correction factor gives the pole and zero directly.

Analysis for the voltage transfer function, or gain, $A \equiv$ $v_{2} / v_{1}$ of the simple lag-lead network of Fig. 14 provides a class practice example. With the capacitance designated the "extra" element $Z \equiv 1 / s C$, students have a choice of whether to take the reference value $Z=\infty$ or $Z=0$; for $Z=\infty$, the reference gain is the zero-frequency gain $A_{0}$, and for $Z=0$ the reference gain is the infinite-frequency gain $A_{\infty}$. In either case, the two driving point resistances are

$$
\begin{aligned}
& Z_{d}=R_{d}=R_{2}+R_{1} \| R_{L} \\
& Z_{n}=R_{n}=R_{2} .
\end{aligned}
$$

Students should be given enough time to draw the circuit under each injection condition, and to understand clearly how these results come about. Those having difficulty can be reminded to visualize the superposition of signals that establishes the nulled output for the null double injection calculation. Once they are confident about these derivations, the result follows immediately by the EET:

$$
A=A_{0} \frac{1+s C R_{n}}{1+s C R_{d}}=A_{\infty} \frac{1+\frac{1}{s C R_{n}}}{1+\frac{1}{s C R_{d}}} .
$$

Other simple examples can easily be invented.

\section{Application of the EET to any Transfer FUNCTION}

The EET may be used to find an extra element correction factor for any transfer function of a linear system. It is necessary merely to identify the "input" and "output" signals; $Z_{d}$ and $Z_{n}$ are then calculated as the driving point impedance seen by the extra element with the "input" zero and with the "output" nulled, respectively. 


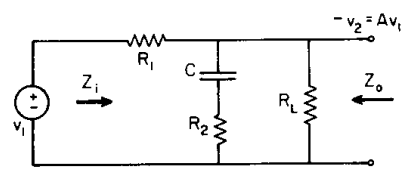

Fig. 14. A lag-lead network.

Some examples of transfer functions are

current drawn from power supply

input voltage

(a transadmittance)

output voltage ripple component

power supply ripple voltage

(a voltage gain; audio susceptibility of a power supply) corresponding driving voltage

any driving current

(a self-impedance, e.g., input or output impedance).

In all cases, the denominator of the transfer function is the "input"' (independent) signal, and the numerator is the "output" (dependent) signal.

Example 4: Input Impedance of the CE Amplifier Stage of Example 1

Consider the amplifier stage of Fig. 5 and its ac smallsignal model of Fig. 6 , in which $C_{1}$ is taken to be a short and $C_{2}$ is omitted. The input impedance is to be determined in the presence of $C_{2}$, identified as an extra element.

At this stage, students can begin doing more of the steps without drawing a separate model for each injection condition. The circuit of Fig. 15 contains the necessary features for all the calculations: the extra element $C_{2}$ is shown, but detached, and the test input signal source can be visualized connected across the terminals to which $C_{2}$ is to be attached.

The "reference transfer function" is the input impedance $Z_{i}=R_{i}$ calculated from the circuit of Fig. 15 without the test signal:

$\left.\left.Z_{i}\right|_{Z=\infty} \equiv \frac{v_{1}}{i_{1}}\right|_{Z=\infty}=R_{i}=R_{s}+R_{B} \|(1+\beta)\left(r_{E}+R\right)$.

Here, $i_{1}$ is the "input" signal, visualized as a current source in Fig. 15, and $v_{1}$ is the "output" signal.

The driving point impedance $Z_{d}$ is found from Fig. 15 with the test signal source visualized as connected across $R$, and with the "input" set to zero. Students are cautioned that zero input in this case means $i_{1}=0$, so the input is open and there is no current in $R_{s}$; hence the result is

$\left.Z_{d} \equiv Z_{d p}\right|_{\substack{\text { input } \\ \text { zero }}}=\left.Z_{d p}\right|_{i 1=0}=R_{d}=R \|\left(r_{E}+\frac{R_{B}}{1+\beta}\right)$.

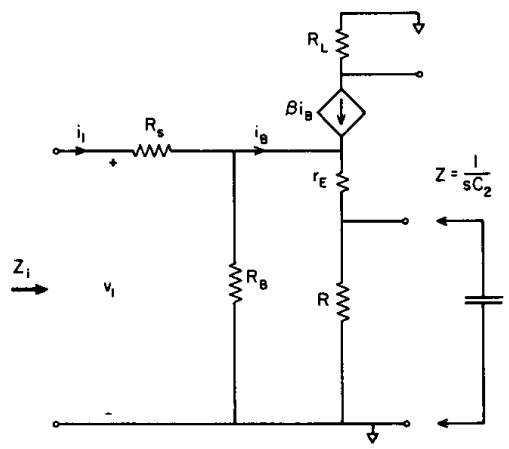

Fig. 15. Small-signal ac model of Fig. 5 for calculation of the input impedance $Z_{i}$ with $C_{1}$ as short and $C_{2}$ considered as an "extra" element.

The driving point impedance $Z_{n}$ is found from Fig. 15 with the test signal source visualized as connected across $R$, and with the "input'" restored and adjusted to null the "output," which in this case is the voltage $v_{1}$. Thus, $R_{s}$ is effectively across $R_{B}$, and the result is

$$
\begin{aligned}
Z_{n} & \left.\equiv Z_{d p}\right|_{\substack{\text { "output" } \\
\text { nulled }}}=\left.Z_{d p}\right|_{\ell^{\prime \prime}=0} \\
& =R_{n}=R \|\left(r_{E}+\frac{R_{s} \| R_{B}}{1+\beta}\right) .
\end{aligned}
$$

The last step is connection of the extra element $C_{2}$ across $R$, and substitution of the above results into the EET:

$$
Z_{i}=R_{i} \frac{1+s C_{2} R_{n}}{1+s C_{2} R_{d}}
$$

Useful Observation 9): For the same circuit and same extra element, the two driving point impedances may be different in the EET correction factor for different transfer functions. In example 4 , the EET for $C_{2}$ applied to the input impedance, $Z_{d}$ and $Z_{n}$ are different from what they were in example 1, the EET for $C_{2}$ applied to the voltage gain of the same circuit. Students should think carefully about why this is so and, in any application of the EET, be sure they properly identify the "input" and "output" signals. In example 4 , they should also think about why $Z_{n}$ for the input impedance is the same as $Z_{d}$ in example 1 for the voltage gain.

Useful Observation 10): In the special case where the transfer function is a self-impedance, setting the input to zero to calculate $Z_{d}$ is the same as opening the input, and nulling the output to calculate $Z_{n}$ is the same as shorting the input. This is because the "output" and "input" are at the same node pair. Note that in this special case the double injection condition is obviated, and the two driving point impedances can be calculated directly from the two equivalent circuits shown in Fig. 16.

Students can do in-class practice on the calculation of the input and output impedances $Z_{i}$ and $Z_{n}$ for the lag-lead circuit of Fig. 14, again with designation of $C$ as an extra element. 


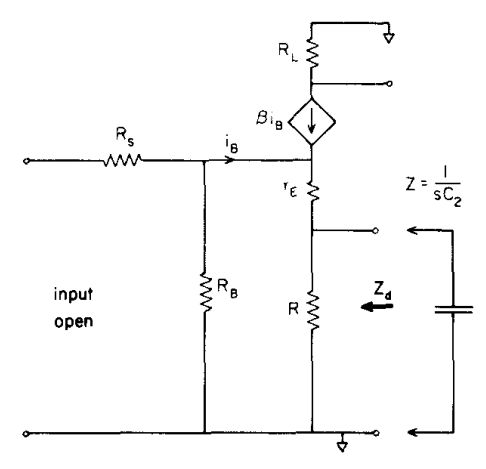

(a)

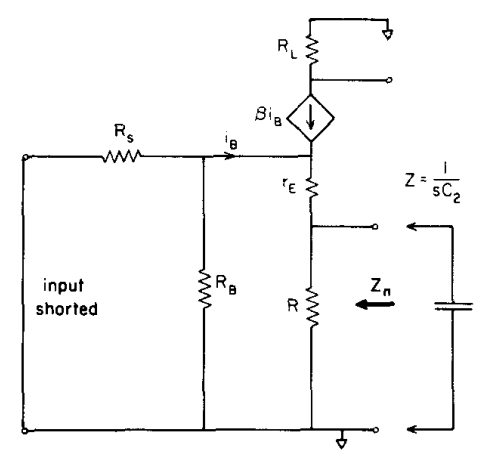

(b)

Fig. 16. Conditions for calculation of the two driving point impedances seen by $C_{2}$ in the model of Fig. 15: (a) for $Z_{d}$, (b) for $Z_{n}$.

VII. Symmetry of the Two Forms of the EET

A useful but implicit feature of the two forms of the EET displayed in (37) and (38) will be introduced in this section by means of an example.

Example 5: Inclusion of Transistor Collector Transition Layer Capacitance $C_{T}$ to $C E$ Amplifier Stage

For the CE amplifier stage of Fig. 5, the midband gain $A_{m}$ has been calculated in example 2 from the model of Fig. 11 as (31). There is nothing in this model that predicts the fall-off of gain at higher frequencies, so one might wish to determine the effect of the transistor collector-base transition layer capacitance $C_{T}$. This can conveniently be done by the EET for an extra parallel element $Z=1 / s C_{T}$ whose reference value is $Z=\infty$ so that the reference gain is the $A_{m}$ already found.

The reference circuit model of Fig. 11 is first simplified by removal of the $R_{s}-R_{B}$ loop by Thevenin's theorem, which leads to the model of Fig. 17. Since $A_{m}$ is already known, the Thevenin voltage generator coefficient is not needed: $v_{1}=0$ for calculation of $Z_{d}$, and $v_{1}$ need not be known for calculation of $Z_{n}$.

For calculation of $Z_{d}$, a test signal is applied as shown in Fig. 18(a) with $v_{1}=0$. In this example, the driving point impedance is not immediately obvious by inspection, so an actual calculation of $Z_{d}=v / i$ is required. This can be done in a variety of ways; the one chosen here starts with choice of the test signal as an independent current source $i$.

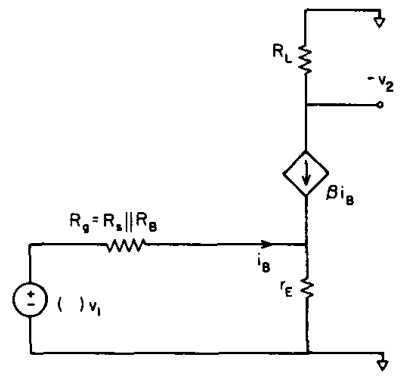

Fig. 17. Reduced small-signal ac model of Fig. 5 for calculation of the gain $v_{2} / v_{1}$ of the common-emitter amplifier stage.

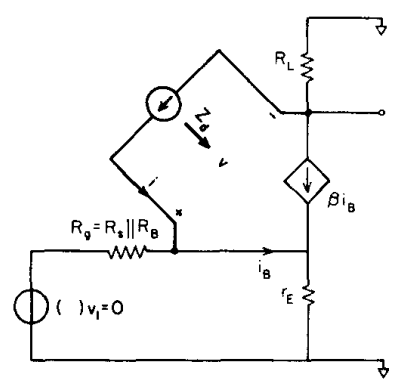

(a)

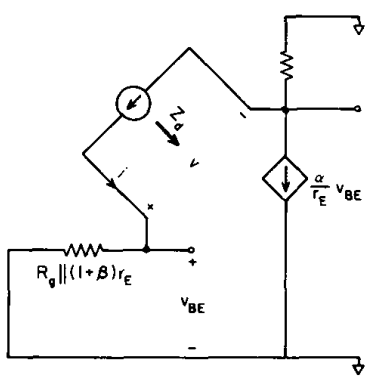

(b)

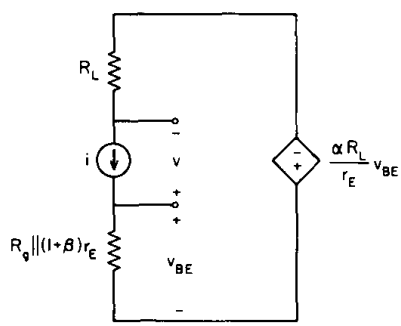

(c)

Fig. 18. (a) Conditions for calculation of $Z_{d}$ in the model of Fig. 17 for the collector transition layer capacitance $C_{T}$ as "extra " element; (b) and (c), successively reduced models.

Successively reduced equivalent circuits are shown in Fig. 18(b) and (c), and since $v_{B E}=\left[R_{y} \|(1+\beta) r_{E}\right] i$, an expression for $Z_{d}$ can be written

$$
\begin{aligned}
\left.\frac{v^{\prime}}{i}\right|_{l^{\prime} \mid=0}= & Z_{d l}=R_{d l}=R_{g} \|(1+\beta) r_{E} \\
& +R_{L}+\frac{\alpha R_{L}}{r_{E}}\left[R_{g} \|(1+\beta) r_{E}\right] .
\end{aligned}
$$


Application of some algebraic force leads to the result in a more compact form:

$$
\begin{aligned}
R_{d} & =\left[R_{g} \|(1+\beta) r_{E}\right]\left(1+\frac{\alpha R_{L}}{r_{E}}\right)+R_{L} \\
& =\left[R_{g} \|(1+\beta) r_{E}\right] R_{L}\left[\frac{1}{R_{L}}+\frac{\alpha}{r_{E}}+\frac{1}{(1+\beta) r_{E}}+\frac{1}{R_{g}}\right] \\
& =\frac{R_{g} \|(1+\beta) r_{E}}{R_{g}\left\|r_{E}\right\| R_{L}} R_{L} .
\end{aligned}
$$

For calculation of $Z_{n}$, the current-source test signal is applied in the presence of $v_{1}$, and imagined adjusted to null the output voltage $v_{2}$. This null double injection condition is shown in Fig. 19. The null propagates according to the following logical sequence of argument. Output voltage $v_{2}=0 \Rightarrow$ current in $R_{L}=0 \Rightarrow$ test signal current source $=$ collector current $\neq 0$, so $i=-\alpha i_{E}$. Also, since $v_{2}=$ $0, v=v_{B E}$.

Hence,

$$
\left.\frac{v}{i}\right|_{i^{\prime}=0}=Z_{n}=R_{n}=-\frac{v_{\mathrm{BE}}}{\alpha i_{E}}=-\frac{r_{E} i_{E}}{\alpha i_{E}}=-\frac{r_{E}}{\alpha} .
$$

The collector capacitance $C_{T}$ can now be added to the model of Fig. 17, and the EET gives the result for the gain as

$$
A=A_{m} \frac{1+s C_{T} R_{n}}{1+s C_{T} R_{d}}=A_{m} \frac{1-s C_{T} r_{E} / \alpha}{1+s C_{T} m R_{L}}
$$

where

$$
m \equiv \frac{R_{g} \|(1+\beta) r_{E}}{R_{g}\left\|r_{E}\right\| R_{L}}
$$

There can be class discussion of the relative values of the pole and zero frequencies, and why the zero is right half-plane. The multiplier $m$ can be related to the Miller effect, and its value as a function of $R_{g}$ can be examined by arranging (55) in the bilinear form

$$
m=\frac{1+\frac{R_{g}}{r_{E} \| R_{L}}}{1+\frac{R_{g}}{(1+\beta) r_{E}}} .
$$

Hence,

$$
\begin{aligned}
& \left.m\right|_{R_{g} \rightarrow 0}=1 \\
& \left.m\right|_{R_{g} \rightarrow \infty}=(1+\beta) \frac{r_{E}+R_{L}}{R_{L}} \approx 1+\beta .
\end{aligned}
$$

This exposes the importance of the value of $R_{g}$ as well as of $R_{L}$ in determining the pole frequency.

In this rather more difficult example of the EET, the simplicity of the calculation of the null double injection calculation for $Z_{n}$ with respect to that of the single injection calculation for $Z_{d}$ is more apparent than in the previous examples, in accordance with useful observation 3 ). Whichever of the numerous methods for finding $Z_{d}$ is used,

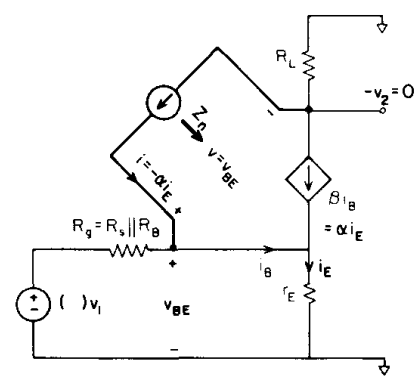

Fig. 19. Conditions for calculation of $Z_{n}$ in the model of Fig. 17 for $C_{T}$ as "extra" element.

it is bound to be fairly complicated because the answer is complicated.

It is the relative difficulty of calculating $Z_{d}$ with respect to $Z_{n}$ that provides the motivation for employing the implicit symmetry feature of the two forms of the EET.

In the derivation of the EET for an extra series element from that for a parallel element, the quantity in the square bracket in (29) was identified as the gain when $Z=0$. This constitutes the symmetry feature

$$
\frac{\left.A\right|_{Z=0}}{\left.A\right|_{Z=\infty}}=\frac{Z_{n}}{Z_{d}}
$$

Alternatively, it is a redundancy relationship, which says that if any three of the quantities are calculated directly from the system model, then the fourth can be found from the redundancy relationship.

In the application of the EET, either $\left.A\right|_{Z=\infty}$ or $\left.A\right|_{Z=0}$ is already known (the reference gain), and $Z_{n}$ is easy to calculate. One therefore has the choice of calculating $Z_{d}$ directly from the circuit model or from the redundancy relationship, whichever is shorter or easier.

\section{Example 5, continued: Alternative Calculation of $Z_{d}$}

It has already been seen that calculation of $Z_{d}$ directly from the circuit model from Fig. 18 required a significant amount of analytic force. To see whether calculation of $Z_{d}$ from (59) is any easier, let us calculate the remaining quantity $\left.A\right|_{Z=0}$.

The appropriate model is shown in Fig. 20. The short between collector and emitter kills the collector current generator, and reduces the model to a simple resistive divider from which

$$
\begin{aligned}
\left.A\right|_{Z=0} & =-\frac{R_{B}}{R_{s}+R_{B}} \frac{r_{E} \| R_{L}}{R_{g}+r_{E} \| R_{L}} \\
& =-\frac{R_{B}}{R_{s}+R_{B}} \frac{R_{g}\left\|r_{E}\right\| R_{L}}{R_{g}} .
\end{aligned}
$$

Hence, by the redundancy relationship (59), with $\left.A\right|_{Z=\infty}$ $=A_{m}$ from (31) and $Z_{n}=R_{n}$ from (53),

$$
\begin{aligned}
Z_{d} & =\frac{r_{E}}{\alpha} \frac{\alpha R_{L}}{r_{E}+R_{g} /(1+\beta)} \frac{R_{g}}{R_{g}\left\|r_{E}\right\| R_{L}} \\
& =\frac{R_{g} \|(1+\beta) r_{E}}{R_{g}\left\|r_{E}\right\| R_{L}} R_{L} .
\end{aligned}
$$




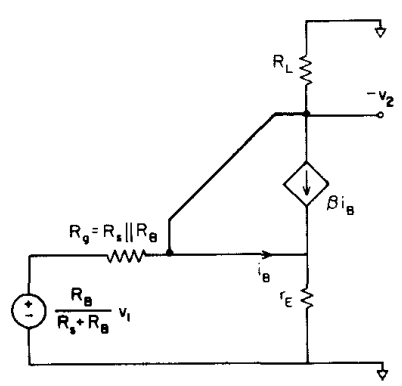

Fig. 20. Model of Fig. 17 with the "extra" element $C_{T}$ shorted.

This derivation requires considerably less effort than the direct calculation leading to (52).

Useful Observation 11): It is often easier to find the other reference gain and to use the redundancy relationship (59) for $Z_{d}$, than to find $Z_{d}$ directly. Note that if in general the reference gains and the null driving point impedance $Z_{n}$ are already known in factored pole-zero form, then $Z_{d}$ is automatically obtained in factored polezero form since no sums or differences of terms appear in the redundancy relationship (59).

\section{The EET for a Dependent Generator}

The EET for the parallel extra element was developed from a sequence of two special cases starting from Fig. 1. If, instead, the second input and output are left unspecified as $u_{i 2}$ and $u_{o 2}$, respectively, the derivation of the theorem can continue in general with $W$ representing any element that connects $u_{02}$ to $u_{i 2}$ externally to the reference system. The two symmetric forms of the result are

$$
A=\left\{\begin{array}{c}
\left.A\right|_{W=0} \frac{1+\left.W \frac{u_{o 2}}{u_{i 2}}\right|_{u_{i 1}=0}}{1+\left.W \frac{u_{o 2}}{u_{i 2}}\right|_{u_{i 1}=0}} \\
1+1 /\left.W \frac{u_{o 2}}{u_{i 2}}\right|_{u_{i 1}=0} \\
\left.A\right|_{W=\infty} \frac{u_{o 2}}{1+1 / W \frac{u_{i 2}}{u_{u_{i 1}=0}}}
\end{array}\right.
$$

together with the redundancy relationship

$$
\frac{\left.A\right|_{W=0}}{\left.A\right|_{W=\infty}}=\frac{\left.\frac{u_{o 2}}{u_{i 2}}\right|_{u_{i 1}=0}}{\left.\frac{u_{o 2}}{u_{i 2}}\right|_{u_{i 1}=0}}
$$

If the $u_{o 2}$ port is different from the $u_{i 2}$ port, $W$ represents a dependent generator at the $u_{i 2}$ port controlled by the signal at the $u_{02}$ port, and $u_{02} / u_{i 2}$ represents the transfer function between the same two ports in the absence of $W$.

The EET for an extra self-impedance $Z$ is easily checked to be a special case this general result, in which the identification of quantities is

$$
\begin{aligned}
\frac{u_{o 2}}{u_{i 2}} \Rightarrow \frac{v_{o 2}}{i_{i 2}} & =\frac{\text { voltage at same port }}{\text { current into port }} \\
& =\text { driving point impedance } \\
& =Z_{d p} .
\end{aligned}
$$

Therefore, the extra element $W$ is identified as

$$
W \Rightarrow Y \equiv \frac{1}{Z}
$$

and

$$
A=\left.A\right|_{\substack{W=0 \\(Z=\infty)}} \frac{1+\left.\frac{1}{Z} Z_{d p}\right|_{u_{o l}=0}}{1+\left.\frac{1}{Z} Z_{d p}\right|_{u_{i \mid=0}}}
$$

which is the EET for an extra parallel impedance $Z$.

The procedure for determination of $u_{o 2} /\left.u_{i 2}\right|_{u_{i 1}=0}$ and $u_{o 2} /\left.u_{i 2}\right|_{u_{01}=0}$ when $W$ represents a dependent generator is as follows.

Identify the (current or voltage) controlling signal (of the dependent generator) as $u_{o 2}$, and the polarity reversed (current or voltage) controlled (dependent generator) signal as $u_{i 2}$. If the dependent generator is originally present, remove it, and replace it with an independent driving signal (of arbitrary impedance, or a voltage source, or a current source, whichever gives the easiest analysis). Determine the transfer functions $u_{o 2} /\left.u_{i 2}\right|_{u_{01}=0}$ and $u_{o 2} /$ $\left.u_{i 2}\right|_{u_{i 1}=0}$.

Example 6. The CE Amplifier Stage with $\beta$ as the Extra Element

Consider the basic CE amplifier stage of Fig. 5 whose midband model is shown in Fig. 17. Identify $W \Rightarrow \beta$ as the extra element, a current-controlled current generator. Identify the controlling signal $i_{B}$ as $\mu_{o 2}$, and the polarity reversed controlled signal $-\beta i_{B}$ as $u_{i 2}$, as shown in Fig. 21 (a). Note that both $u_{i 2}$ and $u_{o 2}$ are currents, in this case. Remove the $\beta$-generator, and replace it with an independent driving signal, as shown in Fig. 21 (b).

Calculation of $u_{02} /\left.u_{i 2}\right|_{u_{i 1}=0}$ from Fig. 22, single injection with $v_{1}=0$, gives

$$
\left.\frac{u_{o 2}}{u_{i 2}}\right|_{u_{i 1}=0}=\frac{r_{E}}{R_{g}+r_{E}} .
$$

Calculation of $u_{o 2} /\left.u_{i 2}\right|_{u_{v 1=0}}$ from Fig. 23, null double injection with $v_{2}=0$, gives

$$
\left.\frac{u_{o 2}}{u_{i 2}}\right|_{u_{o 1}=0}=\infty
$$

because $u_{i 2}=0$ if $v_{2}=0$, but $u_{o 2} \neq 0$.

Since $\left.A\right|_{\beta=0}=0$, only the form of (63) can be used. However, the principal application of the EET for a dependent generator is not to find the gain in the presence 


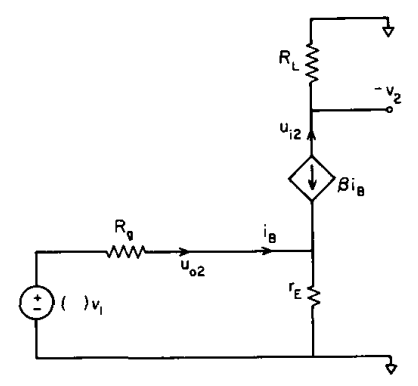

(a)

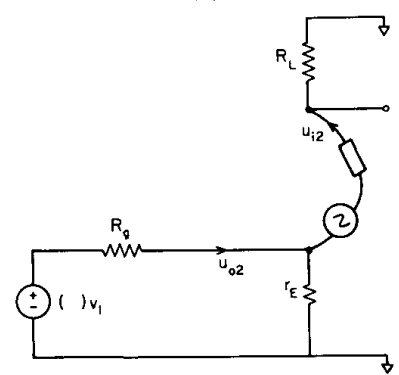

(b)

Fig. 21. (a) Small-signal ac model of Fig. 5 with the $\beta$ dependent generator designated as "extra" element; (b) the "extra" element replaced by an independent generator.

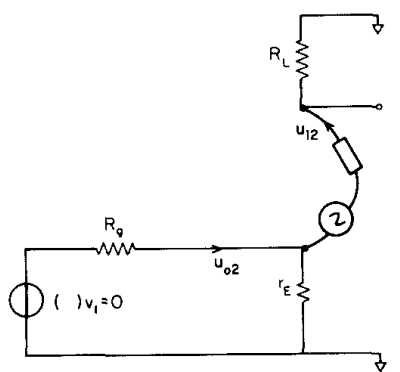

Fig. 22. Conditions in Fig. 21(b) for calculation of $u_{t 2} /\left.u_{i 2}\right|_{u_{11} \ldots 0}$.

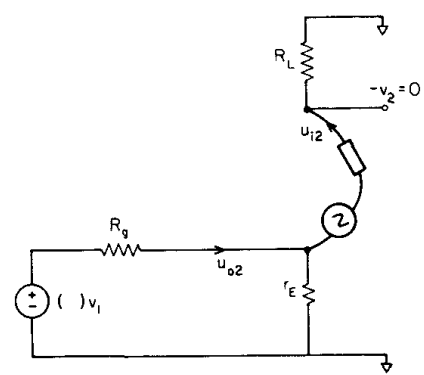

Fig. 23. Conditions in Fig. 21(b) for calculation of $u_{02} /\left.u_{i 2}\right|_{t+11} v^{*}$

of the dependent generator, but to examine the sensitivity of the gain to variations in the value of the dependent generator, as described in the next section.

\section{iX. Return Ratios and Sensitivity}

The EET for a linear system in its general form of (62) and (63) exposes the bilinear dependence of any transfer function $A$ upon any single element $W$ in the system. It is therefore a convenient starting point for finding the sensitivity of $A$ to variations in $W$.
The general EET of (62) through (64) can be written

$$
A=\left\{\begin{array}{l}
\left.A\right|_{W=0} \frac{1+T_{n W}}{1+T_{d W}} \\
\left.A\right|_{W=\infty} \frac{1+\left(1 / T_{n W}\right)}{1+\left(1 / T_{d W}\right)}
\end{array}\right.
$$

with the redundancy relationship

$$
\frac{\left.A\right|_{W=0}}{\left.A\right|_{W=\infty}}=\frac{T_{d W}}{T_{n W}}
$$

where

$$
\left.T_{d W} \equiv W \frac{u_{o 2}}{u_{i 2}}\right|_{u_{i 1}=0}
$$

and

$$
\left.T_{n W} \equiv W \frac{u_{o 2}}{u_{i 2}}\right|_{u_{i 1}=0}
$$

are, respectively, the return ratio and the null return ratio for the element $W$. Note that $T_{d W}$ and $T_{n W}$ are each directly proportional to $W$, and $W$ does not appear elsewhere in the expression for $A$.

The sensitivity $S_{W}^{A}$ of the gain $A$ (or of any transfer function) to the element $W$ can be found as follows. From (68),

$$
\begin{aligned}
\ln A & =\left.\ln A\right|_{W=0}+\ln \left(1+T_{n W}\right)-\ln \left(1+T_{d W}\right) \\
\frac{\Delta A}{A} & =0+\frac{T_{n W} / W}{1+T_{n W}} \Delta W-\frac{T_{d W} / W}{1+T_{d W}} \Delta W .
\end{aligned}
$$

Hence,

$$
\begin{aligned}
S_{W}^{A} \equiv \frac{\Delta A / A}{\Delta W / W} & =\frac{T_{n W}}{1+T_{n W}}-\frac{T_{d W}}{1+T_{d W}} \\
& =\frac{1}{1+T_{d W}}-\frac{1}{1+T_{n W}} .
\end{aligned}
$$

Equation (73) is computationally preferable when both $T_{d W}$ and $T_{n W}$ are small; (74) is preferable when both are large.

Thus, the sensitivity of any transfer function to variations in any element is determined by the return ratio and the null return ratio for that element.

\section{Example 6, continued: Sensitivity of Gain to $\beta$}

For the CE transistor amplifier stage whose midband model is shown in Fig. 17, the two return ratios, from (66), (67) and (71), (72) are

$$
\begin{aligned}
& T_{d W}=\beta \frac{r_{E}}{R_{g}+r_{E}} \\
& T_{n W}=\infty .
\end{aligned}
$$

Hence, from (74),

$$
S_{\beta}^{A}=\frac{1}{1+\frac{\beta r_{E}}{R_{g}+r_{E}}}=\frac{1+\frac{r_{E}}{R_{g}}}{1+(1+\beta) \frac{r_{E}}{R_{g}}} .
$$


It is seen that $S_{\beta}^{A}$ approaches unity if $r_{E} / R_{y} \rightarrow 0$; the fact that it is otherwise less than unity can be ascribed to the "feedback" effect of $r_{E}$.

\section{CONCLUSIONS}

The extra element theorem is really nothing more than a statement of the fact that any transfer function of a linear system is a bilinear function of any constituent element. This was one of the first results given by Bode in his seminal book [1]; specifically, on p. 10. Bode's principal motivation, however, was to study sensitivity properties, and he did not identify the two driving point impedances in the bilinear function.

Whereas the entire edifice of Bode's work is based upon circuit determinants obtained from mesh or nodal analysis, the opposite approach has been taken here. The extra element theorem is developed as an analytical tool, whose motivation is to save work. The result contains only quantities having direct physical interpretation in the system, in particular the two driving point impedances. One unfamiliar concept is needed: null double injection, which has other labor-saving applications, and which is introduced as part of the derivation of the EET in Section II.

The extra element theorem states that any transfer function of a linear system can be expressed in terms of its value when a given "extra" element is absent, and a correction factor involving the given element and two driving point impedances seen by the element. The two versions of the theorem, for the absence of an element corresponding to an infinite impedance value and to a zero value, are displayed in (37) and (38), respectively. The driving point impedance $Z_{d}$ is that seen by the extra element when the system "input" is zero. The driving point impedance $Z_{n}$ is that seen by the extra element when the system "input" is restored and adjusted, in the presence of the second driving signal being used for the driving point impedance determination, to null the system "output." This is the null double injection condition, unfamiliar to students; however, with practice they become convinced that the calculation of $Z_{n}$ is actually easier than that for $Z_{d}$. Circuit analysis methods utilizing driving-point impedances have been emphasized by others [2], [3], although these publications are not readily accessible.

Although the original motivation for the EET was as a method for finding a transfer function in the presence of an extra element in terms of its value before the element was added, which avoids the necessity of reanalyzing the system from scratch, the EET is also useful as a tool to simplify the analysis of a given linear system. A given element can be designated as "extra," removed from the system, and the (simpler) system can be solved for the desired transfer function. By calculation of the two driving point impedances (also for the simpler system), the removed element can be restored by use of the EET. This is particularly easy and useful when the designated "extra" element is a reactance and the simpler "reference" circuit is resistive, since then both driving point impedances are resistances and the EET correction factor gives directly the pole and/or zero resulting from the reactance.
The EET applies to any transfer function of a linear system, not only to a gain but also to a transfer or selfimpedance such as input or output impedance. When applied to an impedance, the EET is equivalent to a form of Blackman's theorem [4]. For a self-impedance, the conditions for calculation of the extra element driving point impedances reduce to an especially simple form: $Z_{d}$ is calculated with the terminals open at which the self-impedance is to be calculated, and $Z_{n}$ is calculated with the same terminals shorted.

In the two forms of the EET given in (37) and (38), there is a redundancy relation given by (59) between the two reference gains and the two driving point impedances. Therefore, whichever of the four parameters is the most difficult to calculate directly can instead be found from the other three. Since only products and quotients are involved in the redundancy relation, use of this alternative does not require refactoring of polynomials in complex frequency to find new poles and zeros. Since one reference gain is usually already known, and since calculation of $Z_{n}$ is always easier than of $Z_{d}$, the choice commonly is between calculation of $Z_{d}$ directly or by calculation of the other reference gain and use of the redundancy relation. The latter turns out to be preferable in the example of Section VII, in which the collector transition layer capacitance is to be added to the model of a transistor amplifier stage.

In Section VIII, the EET is extended to include the case where the extra element is a dependent generator. In this form, the theorem is best suited for calculation of sensitivities.

\section{REFERENCES}

[1] H. W. Bode, Network Analysis and Feedback Amplifier Design. Princeton, NJ: Van Nostrand, 1945.

[2] R. D. Kelly, “Analysis and design of electronic circuits using drivingpoint-impedance technique," Library Copy Media Center. Univ. New Mexico. Albuquerque. NM. Oct. 1984.

[3] J. Choma, Jr., "The systematic analysis of analog electronic circuits." Rep. No. ATR-86 (8140-04)-1, The Aerospace Corporation, El Segundo, CA, Sept. 15, 1986.

[4] R. B. Blackman, "Effect of feedback on impedances," Bell Syst. Tech. J. vol. 22. pp. 269-278, 1943.

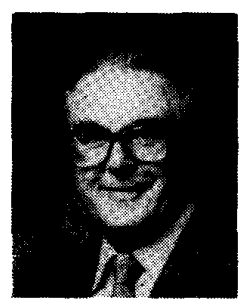

R. D. Middlebrook (S'55-M'56-SM'58-F`78) was born in England on May 16, 1929. He received the B.A. and M.A. degrees from Cambridge University, England, and the M.S. and $\mathrm{Ph} . \mathrm{D}$. degrees from Stanford University. Stanford, CA. in 1952. 1954. 1953. and 1955, respectively.

He is Professor of Electrical Engineering at the California Institute of Technology (Caltech). His publications include numerous papers, a book on solid-state device theory, and another on differential amplifiers. His research interests, formerly in solid-state device modeling, are now in circuits and systems, and particularly in power processing electronics in which he is well-known as author, lecturer, and consultant. $\mathrm{He}$ is especially interested in design-oriented circuit analysis and measure ment techniques which he teaches at Caltech, and he has conducted short courses on his methods in both Europe and the United States.

Dr. Middlebrook was the recipient of the 1982 IEEE William E. Newell Power Electronics Award for Outstanding Achievement in Power Electronics, and a 1982 Award for Excellence in Teaching. presented by the Board of Directors of the Associated Students of Caltech. 